\title{
Health Intelligence Atlas: A Core Tool for Public Health Intelligence
}

\author{
Gabriela M. Wilson ${ }^{1}$ Marion J. Ball ${ }^{1}$ Peter Szczesny ${ }^{2}$ Samuel Haymann ${ }^{2}$ Mark Polyak ${ }^{3}$ \\ Talmage Holmes ${ }^{4}$ John S. Silva ${ }^{1}$
}

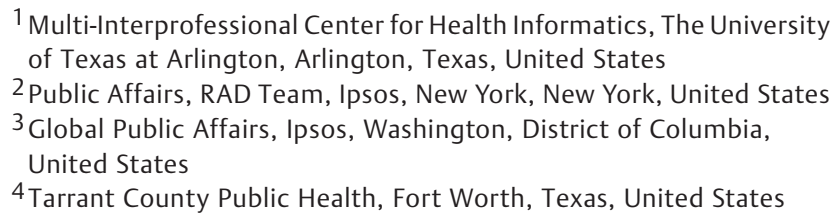

${ }^{4}$ Tarrant County Public Health, Fort Worth, Texas, United States

Address for correspondence Gabriela M. Wilson, PhD, MSc, FHIMSS, SNAI, Multi-Interprofessional Center for Health Informatics, The University of Texas at Arlington, 701 South Nedderman Drive, Arlington, TX 76019, United States (e-mail: gabriela.wilson@uta.edu).

Appl Clin Inform 2021;12:944-953.

\author{
Abstract \\ Keywords \\ - public health \\ - data collection \\ - data visualization \\ - COVID-19 \\ - health informatics \\ - health intelligence \\ - social determinants \\ of health \\ - Social Vulnerability \\ Index \\ - health literacy \\ - geographic \\ information systems
}

Background The dramatic increase in complexity and volume of health data has challenged traditional health systems to deliver useful information to their users. The novel coronavirus disease 2019 (COVID-19) pandemic has further exacerbated this problem and demonstrated the critical need for the 21 st century approach. This approach needs to ingest relevant, diverse data sources, analyze them, and generate appropriate health intelligence products that enable users to take more effective and efficient actions for their specific challenges.

Objectives This article characterizes the Health Intelligence Atlas (HI-Atlas) development and implementation to produce Public Health Intelligence (PHI) that supports identifying and prioritizing high-risk communities by public health authorities. The $\mathrm{HI}-$ Atlas moves from post hoc observations to a proactive model-based approach for preplanning COVID-19 vaccine preparedness, distribution, and assessing the effectiveness of those plans.

Results Details are presented on how the HI-Atlas merged traditional surveillance data with social intelligence multidimensional data streams to produce the next level of health intelligence. Two-model use cases in a large county demonstrate how the HIAtlas produced relevant $\mathrm{PHI}$ to inform public health decision makers to (1) support identification and prioritization of vulnerable communities at risk for COVID-19 spread and vaccine hesitancy, and (2) support the implementation of a generic model for planning equitable COVID-19 vaccine preparedness and distribution.

Conclusion The scalable models of data sources, analyses, and smart hybrid data layer visualizations implemented in the HI-Atlas are the Health Intelligence tools designed to support real-time proactive planning and monitoring for COVID-19 vaccine preparedness and distribution in counties and states. received

March 3, 2021

accepted after revision

August 11, 2021
DOI https://doi.org/

$10.1055 / \mathrm{s}-0041-1735973$.

ISSN 1869-0327. (c) 2021. The Author(s).

This is an open access article published by Thieme under the terms of the Creative Commons Attribution-NonDerivative-NonCommercial-License, permitting copying and reproduction so long as the original work is given appropriate credit. Contents may not be used for commercial purposes, or adapted, remixed, transformed or built upon. (https://creativecommons.org/ licenses/by-nc-nd/4.0/) Georg Thieme Verlag KG, Rüdigerstraße 14, 70469 Stuttgart, Germany 


\section{Background and Significance}

The novel coronavirus disease 2019 (COVID-19) pandemic has highlighted inequities in the health and well-being of vulnerable and marginalized communities and starkly revealed how place, poverty, and place-based sources create racial and ethnic disparities in health and well-being. Community members from disadvantaged backgrounds, including marginalized racial and ethnic groups, have experienced more significant physical, emotional, and economic impacts of the pandemic. ${ }^{1}$ Access to food and income support has become critical to the survival of many families affected by the pandemic, especially those with young children; however, access to these supports has been precarious as services and resource sites have closed, reopened, and changed the format for delivering services to curb the spread of the virus. ${ }^{2}$ Further, the use of public transportation has been reduced to avoid community spread, ${ }^{3}$ diminishing access to the safety net resources in relation to the residence and technological resources of those who depend on them.

Multiple studies on COVID-19 cases and deaths have demonstrated an inequitable burden of the pandemic on low-income and minority communities. These high-risk locations are characterized by clusterings of racial and ethnic minorities, low-income households, unmet medical needs, low health literacy, lack of transportation, and poorer health outcomes. ${ }^{4,5}$ At the same time, the rapid spread of COVID-19 demonstrated that health data and information generated through traditional electronic health record (EHR) systems are insufficient to deliver useful information to clinicians and patients, so they can quickly respond to the crisis. ${ }^{6}$ As the pandemic continued to evolve and new data started being recorded, health information systems were challenged by the lack of "fused" data to support data-driven decisions at national, state, and local levels, as states and counties were responsible for their own COVID-19 response. Several EHR systems have started to include datasets containing social determinants of health (SDOH). However, many public health departments lack access to these types of data. Knowing the locations of the most vulnerable populations and levels of vaccine hesitancies are essential to plan for equitable vaccine preparedness and distribution. This paper provides an overview of a state-of-the-art approach to fuse multiple data sources into health intelligence through the rapid development of the Health Intelligence Atlas (HI-Atlas) prototype. The HI-Atlas fuses multiple data sources and provides visualizations that support a local health department to identify high-risk locations and ensure an equitable vaccination program for all residents.

Map-based planning and monitoring via geographic information system (GIS) is often used to address the challenges described above. However, as the number of GIS layers increases, data layers overlap and obscure the picture. This paper introduces smart hybrid health intelligence layers produced by fusing data streams with logical operations on variables and set "cut-off" points. This approach enables GIS visualization of cooccurrences of variables of interest with one or more smart hybrid layers that focus on extracting the most relevant information without unnecessary data layers, which will make it hard to see the context.

\section{Objectives}

This article describes the development of a prototype, the interactive HI-Atlas. It also describes the set of relevant data sources that can provide the Public Health Intelligence (PHI) to decision makers in communities across the country as they respond to their critical issues during the COVID-19 pandemic. Details are provided below on the relevant data sources and how the HI-Atlas was used to inform public health decision makers and assisted their efforts in two important use cases as follows:

1. Support identification and prioritization of vulnerable communities at risk for COVID-19 spread and vaccine hesitancy.

2. Support the implementation of a generic model for planning equitable COVID-19 vaccine preparedness and distribution.

\section{Methods}

\section{Data Sources}

The various data sources used to identify signals from a multidimensional stream of data are summarized in - Table 1 and described hereinafter.

\section{Centers for Disease Control and Prevention Social Vulnerability Index}

The Centers for Disease Control and Prevention developed the Social Vulnerability Index (SVI) in 2018 for most counties in the United States. ${ }^{7,8}$ SVI indicates the relative vulnerability of every U.S. Census tract, subdivisions of counties for which the Census collects statistical data. SVI ranks the tracts on 15 social factors, including unemployment, minority status, and disability, and groups them into four related themes (i.e., socioeconomic, household composition/disability, minority status/language, and housing type/transportation). Thus, each tract receives a ranking for each Census variable for each of the four themes and an overall ranking. SVI values range from 0 (least vulnerable) to 1 (most vulnerable), or very low (0.0-0.19), low (0.20$0.39)$, moderate $(0.40-0.59)$, high $(0.60-0.79)$, and very high (0.80-1.0). In summary, CDC's SVI classifies as "high-risk neighborhoods," neighborhoods with significant clustering of racial and ethnic minorities, low-income households, and unmet medical needs. ${ }^{9,10}$

\section{Medically Underserved Areas and Populations}

The Medically Underserved Areas (MUAs) are Census tracts designated by the Health Resources and Services Administration as having too few primary care providers, high infant mortality, high poverty, and/or high elderly population. ${ }^{11}$ The Medically Underserved Populations (MUPs) are areas where a specific population group is underserved, including groups with economic, cultural, or linguistic barriers to primary medical care. 
Table 1 Data sources used to identify signals from a multidimensional stream of data

\begin{tabular}{|c|c|}
\hline Domain & Data source \\
\hline \multirow[t]{6}{*}{ Health } & - CDC Social Vulnerability Index (SVI) (https://www.atsdr.cdc.gov/placeandhealth/svi/index.html) \\
\hline & - Medically Underserved Areas and Populations MUAs and MUPs (http://muafind.hrsa.gov/) \\
\hline & - Poverty level data (https://www.census.gov/topics/income-poverty/poverty.html) \\
\hline & $\begin{array}{l}\text { - Health literacy data (https://health.gov/our-work/healthy-people/healthy-people-2030/health-literacy-healthy- } \\
\text { people-2030) }\end{array}$ \\
\hline & - Pharmacy location for vaccine distribution ${ }^{a}$ \\
\hline & $\begin{array}{l}\text { - COVID-19 case counts (https://www.tarrantcounty.com/en/public-health/disease-control-prevention/COVID- } \\
\text { 19.html) }\end{array}$ \\
\hline \multirow[t]{2}{*}{ Access } & - Distance to nearest pharmacy distributing COVID-19 vaccines ${ }^{\mathrm{b}}$ \\
\hline & - Number of persons served by the pharmacy distributing COVID-19 vaccines ${ }^{c}$ \\
\hline \multirow[t]{2}{*}{ Transportation } & $\begin{array}{l}\text { - Transportation data or public transit access (https://nctcoggis.maps.arcgis.com/apps/webappviewer/index. } \\
\text { html?id=cb7420fa96a54b95a6492aeae27075aa) }\end{array}$ \\
\hline & $\begin{array}{l}\text { - Percentage of housing units without vehicles (https://www. } \\
\text { census.gov/programs-surveys/geography/guidance/geo-areas/zctas.html) }\end{array}$ \\
\hline
\end{tabular}

${ }^{\mathrm{a}}$ The Public Health Agency of the county for which the project was conducted.

${ }^{b, c}$ Calculated by the project team (see Pharmacy Distance Calculation section).

\section{Poverty Level Data}

Poverty is a condition in which people or groups lack human needs because they cannot afford them. Poverty is ssociated with various adverse health outcomes, including shorter life expectancy, higher infant mortality rates, higher death rates for the leading causes of death, and access to food and health care. $^{12}$

\section{Health Literacy Data}

Health literacy is the degree to which individuals can find, understand, and use information and services to inform health-related decisions and actions for themselves and others. ${ }^{13}$ Individuals most likely to experience low health literacy including older adults, racial and ethnic minorities, those medically underserved, nonnative speakers of English, and persons with a lower level of education. Factors that affect a person's health literacy skills include education, language, culture, and access to resources. Limited health literacy is associated with lower health outcomes, increased hospitalization rates, decreased use of preventative services, poor health management, and higher costs. National Quartile displays four categories based on the range of scores for the entire United States, with Quartile 1 being the lowest and Quartile 4 as the highest ${ }^{14}$ ( - Table 2).

Table 2 Health literacy categories as defined by the National Quartile $^{11}$

\begin{tabular}{|l|l|}
\hline Category & Score \\
\hline Quartile 1 (the lowest) & 235 or lower \\
\hline Quartile 2 & Higher than 235-247 \\
\hline Quartile 2 & Higher than 247-254 \\
\hline Quartile 4 (the highest) & Higher than 254 \\
\hline
\end{tabular}

\section{Transportation Data}

Transportation and access-related data sources must be included as many residents in these areas have transportation limitations that make getting to vaccination centers problematic The Transit Accessibility Improvement Tool (TAIT) identifies communities that face transportation disadvantages and may have a greater potential need for public transit. The TAIT was developed by the North Central Texas Council of Governments partners with local governments and transportation providers to implement transportation projects throughout the North Central Texas region. It identifies Census block groups with a concentration of residents who are below poverty, living with disabilities, or age 65 years and over. ${ }^{15}$

\section{Percentage of Housing Units Without Vehicles}

Estimated percent of housing units for which no vehicles were available in 2015 to 2019 by the Zip Code Tabulation Area (ZCTA) level. ZCTAs are based on U.S. postal service Zip codes but they are not identical, and in many cases, may be significantly different. Though Zip codes change continuously, ZCTAs are set in 2010 and remain unchanged throughout the decade. Data shown for ZCTAs represent the ZCTA area's information, not the Zip code. ${ }^{16}$

\section{COVID-19 Case Counts}

The cumulative number of COVID-19 cases per Zip code was extracted from the public health website of the project. ${ }^{17}$

\section{Pharmacy Location for Vaccine Distribution}

The list of pharmacies used as vaccine providers was supplied by the public health agency.

\section{Definitions}

- Census tracts are the most used geography by statisticians and policymakers and generally contain between 
1,000 and 8,000 people with an optimum size of 4,000 people.

- Block groups are statistical divisions of Census tracts and generally contain between 600 and 3,000 people. They are a collection of census blocks within a Census tract, sharing the same first digit of their four-digit identifying numbers.

- Blocks are statistical areas bounded by visible features, such as streets, roads, streams, and railroad tracks, and by nonvisible boundaries, such as selected property lines and city, township, school district, and county limits and short-line-of-sight extensions of streets and roads.

\section{Health Intelligence Atlas Structure}

The HI-Atlas prototype is a functional and interactive dashboard built within ArcGIS, a GIS for working with maps, geographic information, and data. ${ }^{18}$ The prototype is available to the public health agency and our staff and allows the users to define specific parameters for selected data sources, filter selections, and visualize smart hybrid data across geospatial boundaries, serving the two objectives described hereinafter.

\section{Support Identification and Prioritization of Vulnerable Communities at Risk for COVID-19 Spread and Vaccine Hesitancy}

Measures of SDOH (transportation, access to health services, income, etc.) were needed to help identify correlations with living in high-risk, impoverished neighborhoods, as the large county selected as initial implementation has a poverty rate at $21 \%$ and higher, minimal health literacy, ${ }^{19,20}$ and a substantial digital divide. The at-risk Census tracts were identified using the $\mathrm{CDC}^{21} \mathrm{SVI},{ }^{9,10}$ generated to identify populations that are especially at risk during public health emergencies because of socioeconomic status, household composition, minority status, housing type, and transportation. Of particular interests were the relationships between COVID-19 cases and individual subcomponents of the SVI, including socioeconomic status, household composition, disability, minority status and language, and housing type and transportation. Some of those were further explored with such characteristics as poverty levels, populations of more than 65 years, and percentage of minorities being analyzed on a Zip code level and compared with the spread of the disease. Also included were the health care literacy, MUA, and MUP data, which-just like SVI-aggregated on a Zip code level to allow for comparisons with Covid-19 case counts.

Once the data files were ingested from respective sources, the process of geomatching began depending on the data source being used. Some of the information was provided at a Zip code, Census tract, and census block group levels, with the CDC SVI data serving as the anchoring point at the Census tract level. A unified data template and a "dictionary" or "cross-walk" were created to allow future aggregations. Data were cleaned, removing data points that were outside of the borders of the county that was part of this project. At the same time, to be able to perform the analysis of COVID-19 cases, available only at the Zip code level, populations had to be aggregated at a Zip code level with the values of individual components (e.g., socioeconomic status, minority status, and language, household composition, and others) being weighted on the population of a given Census tract. A similar approach was taken when incorporating MUA/the Population and Healthcare Literacy data inputs.

\section{Support Implementation of a Generic Model for Planning Equitable COVID-19 Vaccine Preparedness, Distribution, and Assessment}

As a result of the analysis presented in the previous objective, an interactive dashboard has been constructed within ArcGIS, allowing users to understand better possible challenges with equitable and efficient distribution of the COVID-19 vaccine. The dashboard consists of two types of maps, individual layers and smart hybrid layers.

\section{Individual Layers}

- Individual facilities (i.e., pharmacies listed as vaccine providers) that allow users to identify vaccine distribution locations.

- CDC's SVI, health care literacy, households without cars, and MUAs and MUPs. These data streams, combined with facilities, allowing users to identify vulnerable census block groups and tracts where potential mobile vaccination services may need to be deployed.

- Distances for walking (20 and 40 minutes) and driving ( 5 minutes) have been calculated for all pharmacies across the county, allowing users to identify areas beyond local residents' convenient reach. ArcGIS application was used to fuse the previously described tabular data with such elements as locations of pharmacies, etc. The "Create Drive-Time Areas" tool was utilized to identify the areas that could be reached within a specified travel time or travel distance along a street network based on travel mode.

\section{Smart Hybrid Layers}

To improve the functionality of the dashboard, several smart hybrid layers have been created utilizing ArcGIS's data exploration tools and custom data sources used for the individual layers.

- Vulnerability overlaps: the SVI index was combined with health care literacy and underserved areas datasets. Census tracts were then color-coded based on the number of present challenges. Two different versions of this layer have been created to increase the granularity of insights, one using the 0.6 SVI threshold and another using 0.8 as the indicator of social vulnerability.

- Vulnerability plus pharmacy per capita: separately, SVI was combined with population counts, allowing users to identify vulnerable areas without pharmacies, vulnerable areas with more than 7,000 residents per pharmacy, and areas of any vulnerability but with more than 7,000 local residents per pharmacy.

The project team met with public health staff biweekly to establish the variables of interest, review the resulting maps 
and layers, and provided feedback for the next iteration. Compared with traditional GIS layers that tend to obscure details, the public health staff found the hybrid layers accessible through HI-Atlas extremely useful in providing the necessary visualizations of cooccurrences, substantially more informative and easier to use. As the scope of the project was to develop a rapid prototype, there was no formal validation performed. The public health staff provided insights into the utility and quality of the hybrid layers at each iteration.

\section{Pharmacy Distance Calculations}

Distances to the nearest pharmacy were calculated using actual walking and driving distances based on the shortest route on a street network as provided by the geoprocessing service ShortestRouteService via ArcMap 10.3. ${ }^{18}$ Census block groups were then classified based on that distance into the following categories: 0 to 2 miles away (translating to $\sim 0-40$ minutes of walk, using the average human walking speed of 3.1 miles per hour), 2 to 4 miles away ( $\sim 40-80$ minutes), and +4 miles (over 80 minutes away).

Spatial data were analyzed using spatial regression, spatial lag models, spatial autocorrelation (clustering), street and transit path network models to evaluate the distance between pharmacies listed as vaccine providers and socially vulnerable census block groups, Census tracts, and Zip codes. The analysis involved determining relationships between distances to pharmacies overlaid with SVI and health care literacy at different geographic classifications in the county studied in this project.

\section{Results}

The implementation of the HI-Atlas produced PHI that was used to support two exemplar use cases in the project county as mentioned below:

1. Support identification and prioritization of vulnerable communities at risk for COVID-19 spread and vaccine hesitancies

2. Support implementation of a model for an equitable and rapid COVID-19 vaccine preparedness and distribution campaign.

These use cases and the generic model for vaccine preparedness and distribution were developed, so that the PHI produced for the project county could be quickly implemented for other counties in each state, using readily available local and national data sources. The data-driven process for identifying, prioritizing, and implementing a COVID-19 vaccine preparedness and distribution is shown in - Fig. 1. Based on criteria established by public health staff, the set of variables, and their cut-off points, the HI-Atlas would enable the public health staff to generate a list of locations that met their criteria by selecting locations and developing a report of the most vulnerable areas at the Census tract level. The public health staff would then prioritize those locations. The next step would be to analyze the health literacy levels for each selected Census tract and determine if that Census tract

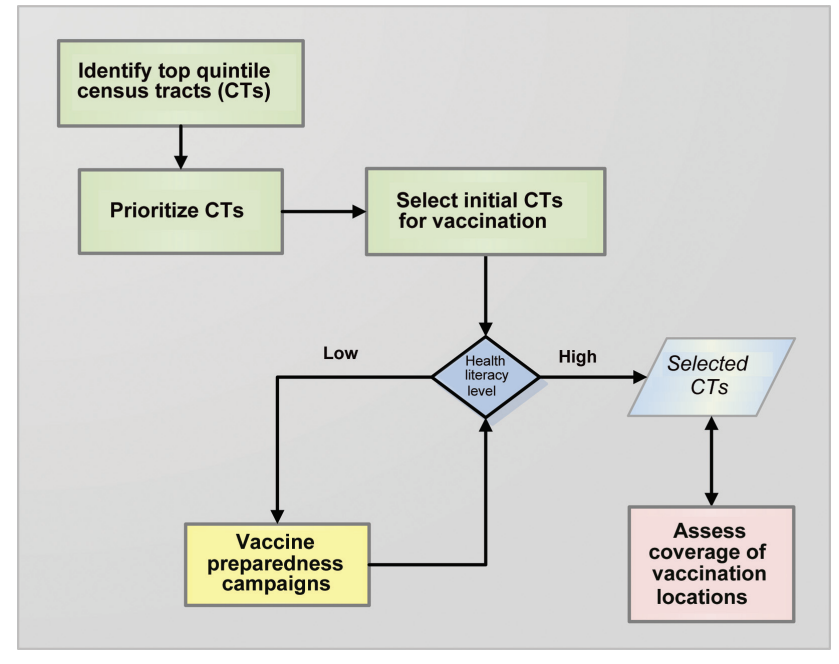

Fig. 1 Data-driven decision tree for vaccine preparedness and distribution.

needs to have a vaccine preparedness campaign to help address any vaccine hesitancies. In addition, the public health staff can assess the need for mobile vaccine vans and pop-up vaccination locations if they determine that transportation is problematic for a given Census tract (i.e., many elderly persons, households without transportation, and others). Specific examples of the PHI that supports datadriven decisions for vaccination preparedness and distribution campaigns are provided within each project's objectives.

\section{Support Identification and Prioritization of Vulnerable Communities at Risk for COVID-19 Spread and Vaccine Hesitancies}

The initial action in our model uses the HI-Atlas and the CDC SVI to identify vulnerable communities and their locations within the project county ( - Fig. 2A). The dark red areas identify the locations of the 5th quintile Census tracts (SVI $>0.8$ ) in the project county based on the CDC SVI. These areas overlap with MUAs ( - Fig. 2B) and are also associated with higher cumulative COVID-19 cases (-Fig. 2C).

Next, as indicated in -Fig. 1, the HI-Atlas supported prioritization of the highest risk areas by ranking Census tracts SVIs within the 5th quintile from the most vulnerable (i.e., the highest $\mathrm{SVI}$ ) to lowest (i.e., $\mathrm{SVI}>0.8$ ) but still classified as high-risk areas. However, not all census tracts have the same health literacy levels, ethnicity, and poverty levels. Areas with higher health literacy would be more likely to accept vaccination. ${ }^{22,23}$ In areas with lower health literacy levels, vaccine preparedness campaigns will be needed to raise COVID-19 and vaccine awareness levels.

HI-Atlas then supported classifying Census tracts into two groups; those census tracts with higher health literacy levels, ready for vaccinations, and those needing vaccine preparedness before vaccination efforts ( - Fig. 1). As indicated in - Fig. 3A and B, high vulnerable areas and several block groups with low health literacy levels were identified, placing them between the first and second quartiles at the national, state, and National Assessment of Adult Literacy (NAAL) levels. ${ }^{14}$ The yellow circled areas in $~-F i g .3 B$ highlight 

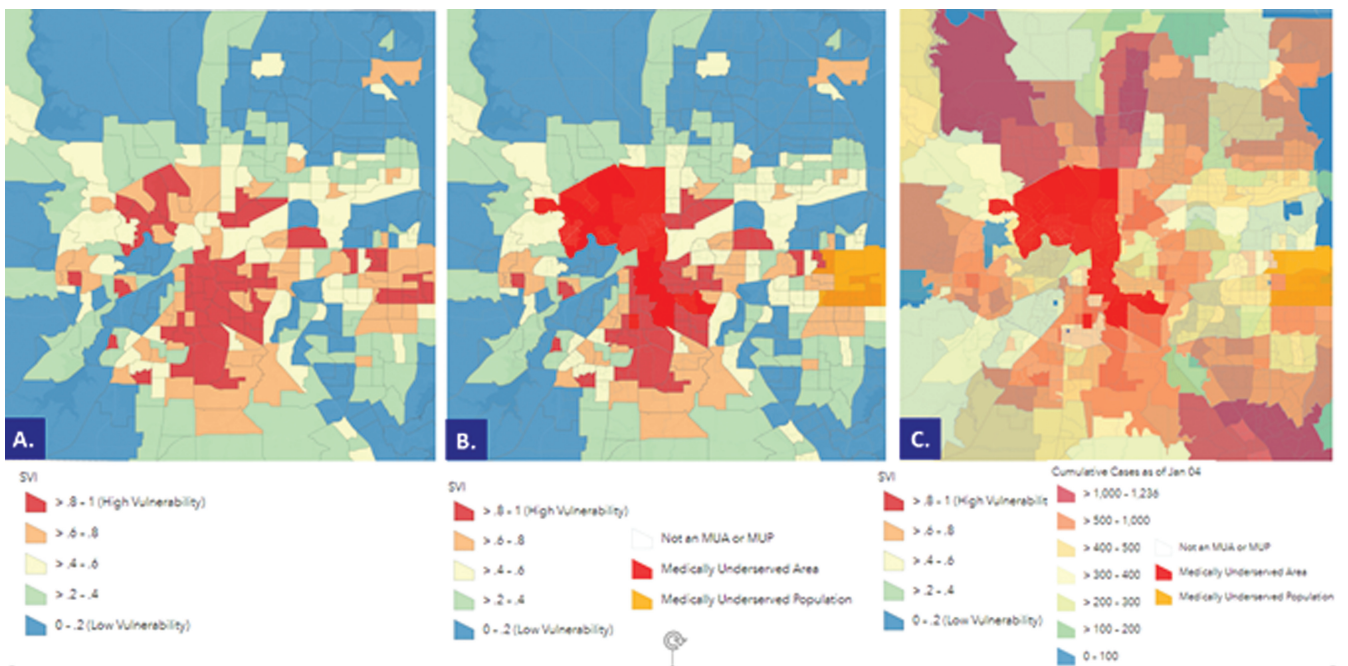

Fig. 2 Color-coded SVI Census tracts (CTs), Medically Underserved Areas (MUAs), and Medically Underserved Populations (MUPs), along with cumulative COVID-19 cases (as of Jan.4, 2021) within the project county. (A) Color-coded SVI census tracts; areas in dark red depict CTs with an SVI $>0.8$, the county's highest vulnerability locations. (B) Color-coded SVI census tracts overlaid with MUAs and MUPs. Areas in bright red depict MUAs. (C) Color-coded SVI census tracts overlaid with MUAs, MUPs, and cumulative COVID-19 cases. Areas in dark pink and light pink represent areas with the largest number of cumulative COVID-19 cases within the project county. COVID-19, novel coronavirus disease 2019; SVI, Social Vulnerability Index.
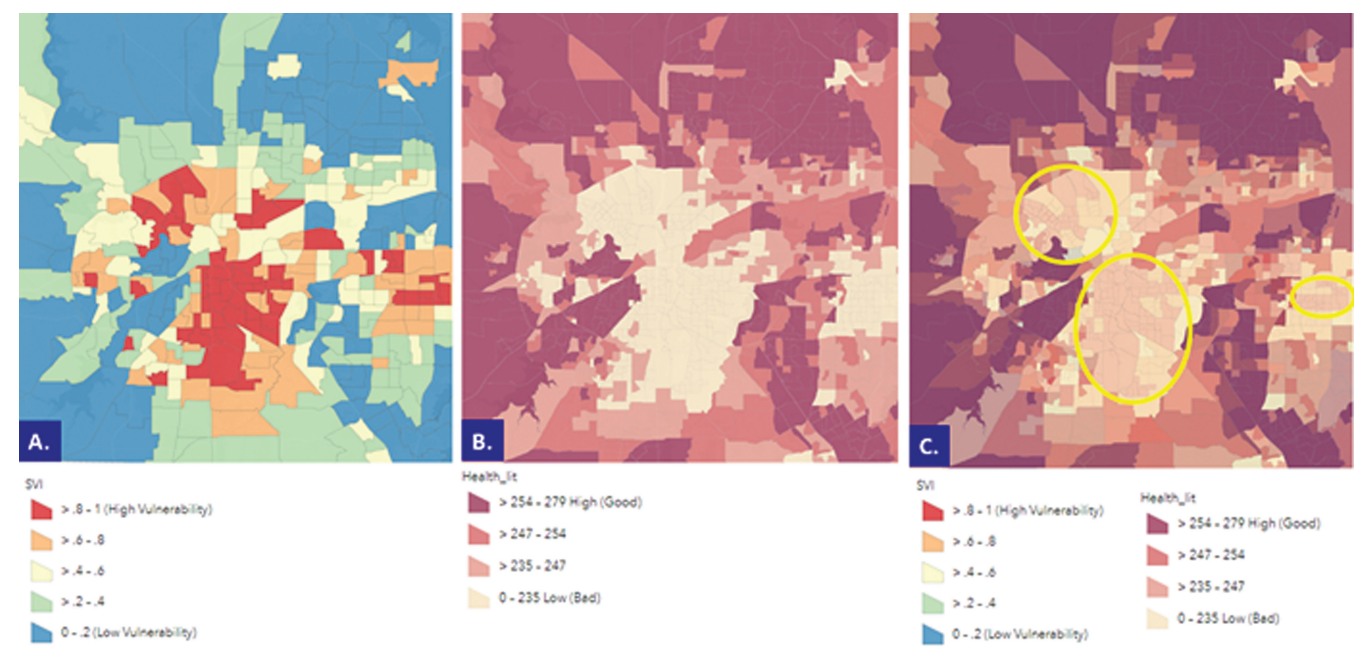

Fig. 3 Color-coded CDC SVI census tracts and health literacy levels within the project county. (A) Color-coded CDC SVI census tracts; areas in dark red depict Census tracts (CTs) with an SVI $>0.8$, the county's highest vulnerability locations. (B) Color-coded health literacy levels within the project county. Areas in light yellow represent areas within the National Quartile 1 (i.e., lowest literacy level). (C) Color-coded CDC SVI census tracts and health literacy levels within the project county. Yellow circled areas are highly vulnerable areas (dark red) where health literacy is low (light yellow). CDC, Centers for Disease Control and Prevention; SVI, Social Vulnerability Index.

areas with the lowest health literacy located in the most vulnerable locations (SVI $>0.8$ ).

\section{Support Implementation of a Model for an Equitable and Rapid COVID-19 Vaccine Preparedness and Distribution}

Concurrent with vaccination efforts in counties ready to receive the COVID-19 vaccine, the project county would initiate vaccine preparedness in those high-risk census tracks with the lowest health literacy levels. As indicated in - Fig. 4A, these neighborhoods consist primarily of minorities and are classified as MUAs and MUPs with poorer health outcomes. (-Fig.4B). This latter figure introduces smart hybrid health intelligence layers, produced by fusing the three data streams where there are cooccurrences of high SVI and low health literacy and MUAs. The bright red areas in - Fig. $4 B$ represent areas where all three of these occur together and identify areas that are, potentially, the most challenging locations for both vaccine preparedness and vaccination. Compare the clarity of - Fig. 4B in which only areas of interest are displayed with - Fig. $3 \mathrm{C}$ in which both layers are being displayed. These factors, combined with vaccine misinformation, disinformation, and mistrust, play a significant role in vaccine hesitancy in minorities and the inherent mistrust toward decision makers. ${ }^{24}$ Knowing the racial and ethnic composition of these neighborhoods, health 


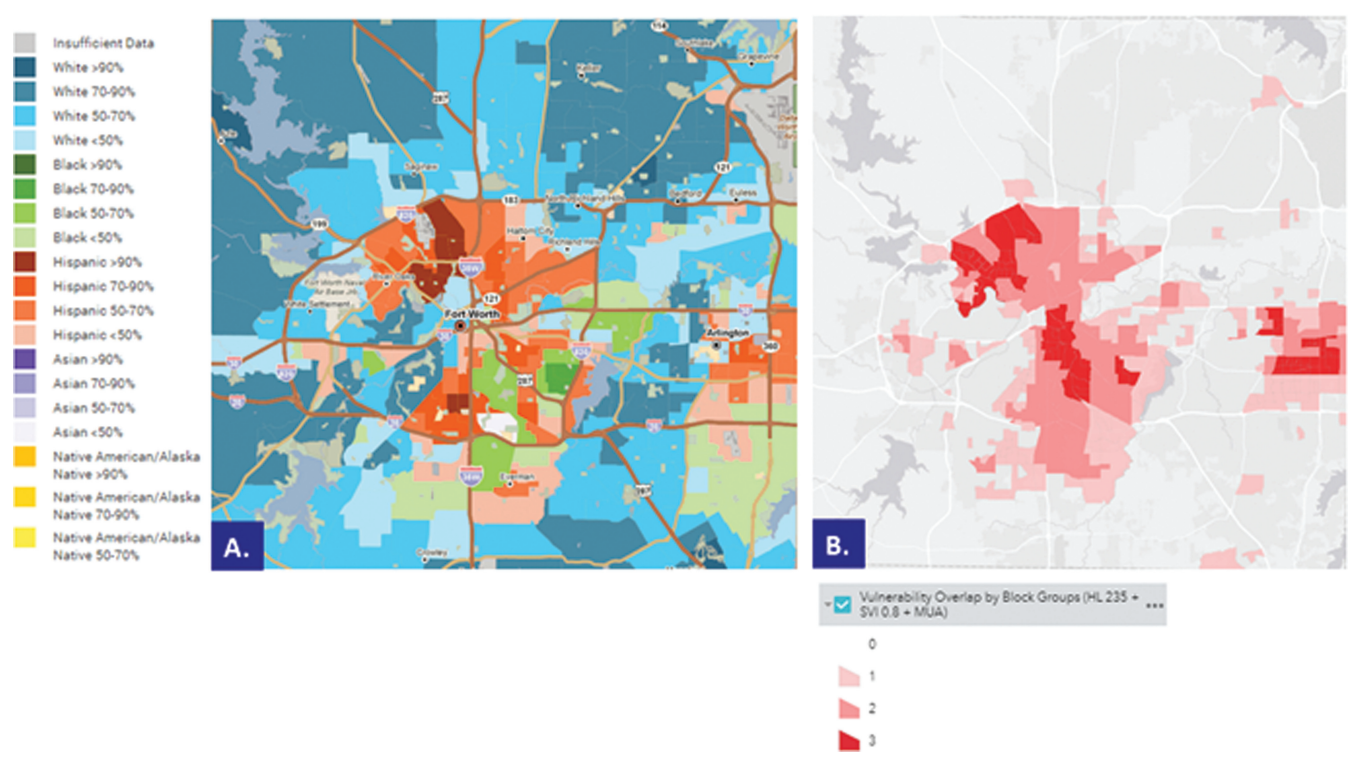

Fig. 4 Color-coded racial and ethnic groups, SVI census tracts, low health literacy level areas, and Medically Underserved Areas (MUAs), and Medically Underserved Populations (MUPs) within the project county. (A) Color-coded percentage of predominant racial or ethnic groups (PolicyMap LLC, Philadelphia, Pennsylvania). ${ }^{29}$ (B) Color-coded SVI $>0.8$ census tracts with low health literacy levels (0-235, National Quartile 1 ), and MUAs and MUPs within the project county. SVI, Social Vulnerability Index.

literacy levels, and MUPs' location are crucial for successful vaccine preparedness planning and campaigns.

HI-Atlas serves as a conduit toward delivering the PHI needed to prepare communities with culturally sensitive COVID-19 health and vaccine information, specifically aligned with the community that addresses issues of vaccine hesitancy, trust, and corrects misinformation about vaccination. As each community completes its COVID-19 preparedness activities, it can then confidently move into the vaccination campaign. Similarly, as each county has developed its own plans for vaccination locations, it is essential to know how those locations will be staffed, how vaccine doses would be distributed to the sites, and how they would be administered and reported. The project county decided to use local pharmacies to provide vaccinations to the public and supplement these with mobile vaccination units. The HIAtlas provided smart hybrid layer visualizations that combined pharmacy locations with census tracts with SVI $>0.6$ and no pharmacy (see red shaded areas in -Fig. 5) and census tracts with $\mathrm{SVI}>0.6$ with pharmacies greater than 7,000 residents (see yellow shaded areas in - Fig. 5). These health intelligence visualizations enabled the public health officials to identify areas with high risk and insufficient access to vaccine distribution centers.

Public health decision makers sought PHI to identify areas where residents have transportation challenges in addition to those without vaccination locations. The HI-Atlas provided hybrid layer visualizations for households without cars ( - Fig. 6A) and walking (-Fig. 6B) and driving times ( - Fig. 6C) to the nearest pharmacy. These displays helped public health staff identify locations where residents did not have equitable access to vaccination locations. As new vaccination locations are being established, the updated list of providers can be easily uploaded into the HI-Atlas to reassess vaccination coverage.

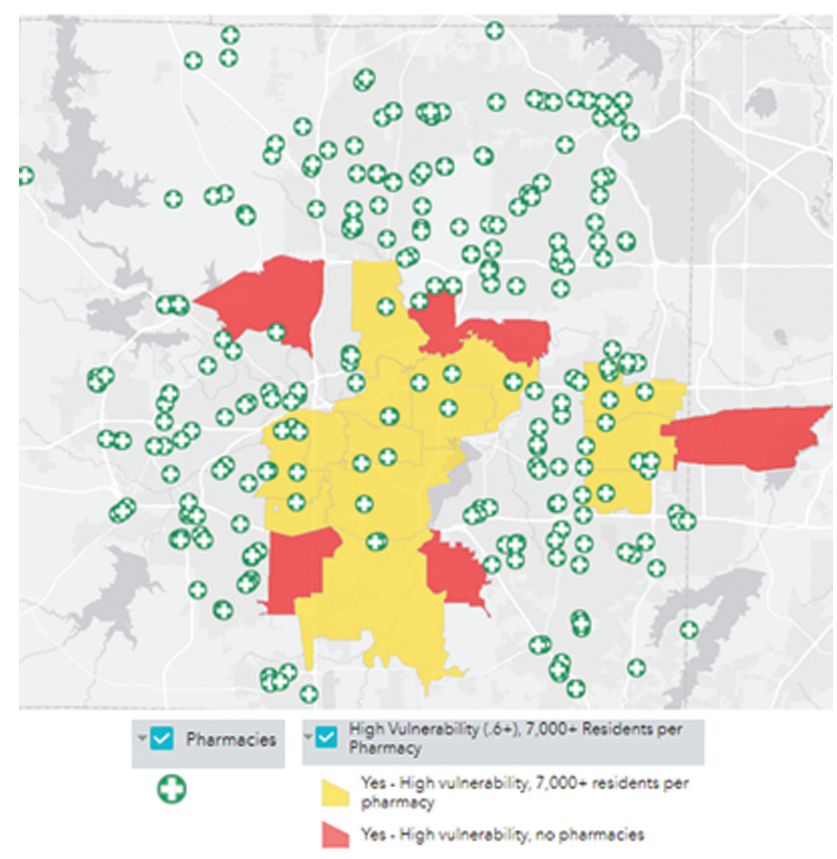

Fig. 5 Smart hybrid layers with census tracts with $\mathrm{SVI}>0.6$ and no pharmacy (red) or pharmacy serving more than 7,000 residents (yellow). SVI, Social Vulnerability Index.

\section{Discussion}

The COVID-19 pandemic has altered what we thought was "normal." As the number of cases continued to rise exponentially, there was significant pressure on public health authorities to quickly and effectively respond. Multiple dashboards were created to report the number of active cases and deaths at the state, county, school district levels, etc. These post hoc catalogs have proved beneficial to identifying those "hotspot" locations with the most COVID-19 cases and deaths. 


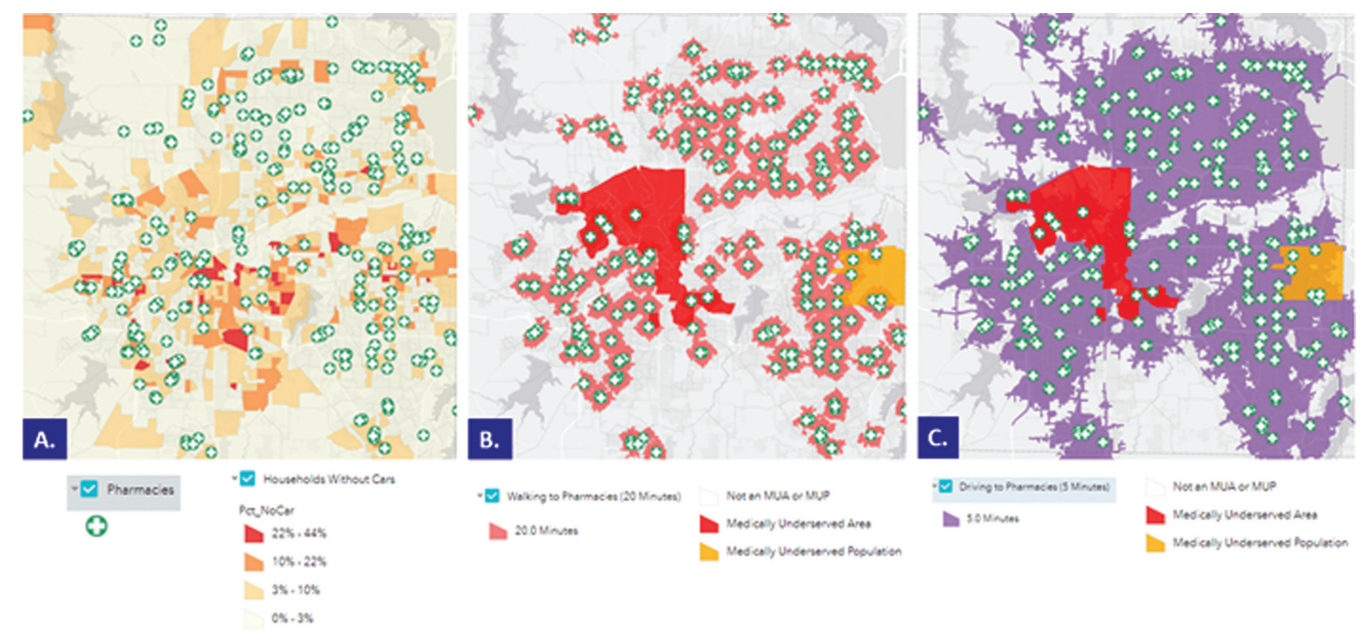

Fig. 6 Locations of pharmacies listed as vaccine providers and transportation accessibility/availability to these locations. (A) Locations of pharmacies and percentage of households without cars. (B) Walking distance to the nearest pharmacy under 20 minutes. (C) Driving distance to the nearest pharmacy under 5 minutes. MUAs and MUPs areas are displayed in red and yellow. MUA, medically underserved areas; MUP, medically underserved populations.

However, examining data visualizations about COVID-19 highlights three ways that these dashboards can mislead viewers as follows: (1) by displaying inadequate data, (2) by manipulating scales and visual distance, and (3) by omitting contextual labels needed to understand a chart's message entirely. ${ }^{25}$

It is apparent that these dashboards alone are insufficient to provide real-time insights for decision makers. One year after the first case of COVID-19, states and counties have been challenged to find fast ways of distributing vaccines to vaccination locations, and public health officials have been working tirelessly to provide equitable vaccine distributions and identify the areas with a high level of vaccine hesitancies. Very recently, to support state and local communication and outreach efforts, the Office of the Assistant Secretary for Planning and Evaluation (ASPE) developed state, county, and substate level predictions of hesitancy rates using the most recently available federal survey data. This interactive map shows estimates of the percent of the population in each county that may be vaccine hesitant. ${ }^{26}$ This map's limitation is that it depends on how each state provides the vaccination rate information. For example, Texas vaccination rate information includes aggregated data at the state level and cannot be stratified by county. Therefore, the approach presented in this article is unique, making HI-Atlas an extremely useful tool to public health officials. It focuses on exploiting existing capabilities to ingest relevant, diverse data sources, analyze them, and generate appropriate health intelligence products that enable users to take more effective and efficient actions for vaccination preparedness and vaccine distribution challenges. HI-Atlas, was developed and is being used to produce map-based (GIS) health intelligence that supported public health and local and state agencies to plan for and conduct vaccination campaigns. One of the challenges with GIS is that as layers are added to a view, the intelligence can be obscured by the overlap of these multiple data streams. As a clear example, =Fig. 3 C depicts two overlapping layers, one set of shades for SVI quintiles and another set of shades for health literacy quartiles. As users were interested in visualizing the areas with low health literacy values within Census tracts with high SVI (i.e., SVI $>0.8$ ), a smart hybrid layer was created. This layer depicted the health literacy levels, MUAs, and MUPs for those Census blocks that had the highest vulnerability. This view helped identify those areas that needed COVID-19 vaccine preparedness before vaccinating people living in these locations. HI-Atlas was also used to highlight the areas that needed additional mobile vaccine distribution units due to the lack of vaccine providers and transportation in high-risk areas. The benefit of utilizing smart hybrid layers enables decision makers to focus on the most relevant data and respond based on community's needs. Nevertheless, data alone cannot reduce or eliminate inequalities. Examining the location and accessibility to resources are critical to community members from disadvantaged backgrounds, including marginalized racial and ethnic groups. Understanding the spatial distribution of health and welfare resources in relation to the location of where resources are needed in the daily lives of vulnerable families provides opportunities to improve access to resources, which can be more readily identified and incorporated into high-impact strategies for policy implementation and service delivery. However, these types of priorities generally include little input from service users, ${ }^{27}$ leading to gaps in geographic and practical barriers to resources. ${ }^{28}$ Without a clear understanding of how community members from disadvantaged backgrounds, including marginalized racial and ethnic groups, have been unequally affected by the pandemic, we cannot fix the social and racial injustice and inequity that is at the forefront of public health. To achieve health equity, communities need to be involved in system design, and SDOH need to be grounded in principles of equitable evaluation in terms of what policy, practice, and planning mechanisms of service location, and access and delivery could be modified to increase resource acquisition and stabilization among low income, marginalized families living in under-resourced areas. 


\section{Conclusion}

The COVID-19 pandemic demonstrated the need for hybrid surveillance systems that can merge traditional surveillance data with multidimensional data from search queries to produce the next level of health intelligence. This approach is unique. It uses smart hybrid health intelligence layers that enable the extraction of the most relevant information designed to support real-time proactive planning and monitoring for COVID-19 vaccine preparedness and distribution.

\section{Clinical Relevance Statement}

The Health Intelligence Atlas (HI-Atlas) assists public health officials with the data-driven health intelligence for COVID-19 vaccine preparedness and delivery at the county and state levels.

\section{Multiple Choice Questions}

1. What was the main reason for developing the Health Intelligence Atlas (HI-Atlas)?

a. To visualize COVID-19 active case data

b. To facilitate aggregation of data generated by health care systems

c. To support health care workers through reliable information

d. To support identifying and prioritizing high-risk communities by public health authorities

Correct Answer: The correct answer is option d. The main reason for developing the HI-Atlas was to produce Public Health Intelligence (PHI) that supports identifying and prioritizing high-risk communities by public health authorities.

2. What is the advantage of using smart hybrid health intelligence layers within HI-Intel Atlas?

a. It allows the addition of geospatial data

b. Data are being added automatically

c. Enables GIS visualization of co-occurrences of variables of interest

d. Allows for automatic interpretation of data

Correct Answer: The correct answer is option c.The advantage of using smart hybrid health intelligence layers is that it enables geographic information system (GIS) visualization of cooccurrences of variables of interest by extracting the most relevant information without unnecessary data layers which will make it hard to see the context.

Protection of Human and Animal Subjects

This work was reviewed by the Institutional Review Board and concluded it was not humans subjects research.

\section{Funding}

This project was funded from institutional money. No additional funding was received from external sources.
Conflict of Interest

None declared.

\section{References}

1 Centers for Disease Control and Prevention. COVID-19 in Racial and Ethnic Minority Groups. Accessed August 25, 2021 at: https://www.hsdl.org/?view\&did=837299

2 Agostino D, Arnaboldi M, Lema MD. New development: COVID-19 as an accelerator of digital transformation in public service delivery. Public Money Manag 2021;41(01):69-72

3 Liu L, Miller HJ, Scheff J. The impacts of COVID-19 pandemic on public transit demand in the United States. PLoS One 2020;15 (11):e0242476

4 Karmakar M, Lantz PM, Tipirneni R. Association of social and demographic factors with COVID-19 incidence and death rates in the US. JAMA Netw Open 2021;4(01):e2036462

5 Poulos J, Zhu L, Shah AD. Data gaps in electronic health record (EHR) systems: An audit of problem list completeness during the COVID-19 pandemic. Int J Med Inform 2021; 150:104452

6 Health equity considerations and racial and ethnic minority groups. Accessed August 25, 2021 at: https://asprtracie.hhs. gov/technical-resources/resource/9138/health-equity-considerations-and-racial-and-ethnic-minority-groups

7 Flanagan BE, Gregory EW, Hallisey EJ, Heitgerd JL, Lewis B. A social vulnerability index for disaster management. J Homel Secur Emerg Manag 2011;8(01):1-24

8 Gaynor TS, Wilson ME. Social vulnerability and equity: the disproportionate impact of COVID-19. Public Adm Rev 2020;80 (05):832-838

9 CDC/ATSRD social vulnerability index. Accessed August 25, 2021 at: https://www.atsdr.cdc.gov/placeandhealth/svi/index.html

10 CDC/ATSRD SVI data and documentation download. Accessed February 20, 2021 at: https://www.atsdr.cdc.gov/placeandhealth/svi/data_documentation_download.html

11 Health Resources \& Services Administration. MUA find. Accessed August 25, 2021 at: http://muafind.hrsa.gov/

12 United States Cencus Bureau Poverty. Accessed August 25, 2021 at: https://www.census.gov/topics/income-poverty/poverty.html

13 US Department of Health and Human Service. Health literacy in healthy people 2030. Accessed August 25, 2021 at: https:/l health.gov/our-work/healthy-people/healthy-people-2030/ health-literacy-healthy-people-2030

14 University of North Carolina at Chapel Hill. Health literacy data map. Accessed August 25, 2021 at: http://healthliteracymap.unc. edu/

15 North Central Texas Council of Governments. Transit accessibility improvement tool. https://nctcoggis.maps.arcgis.com/apps/webappviewer/index.html?id=cb7420fa96a54b95a6492aeae27075aa Accessed August 25, 2021

16 The United States Census Bureau. ZIP code tabulation areas (ZCTAs). https://www.census.gov/programs-surveys/geography/guidance/geo-areas/zctas.html Accessed August 25, 2021

17 Coronavirus (COVID-19) inTarrant County. Accessed August 25, 2021 at: https://www.tarrantcounty.com/en/public-health/disease-control-prevention/COVID-19.html

18 ArcGIS. ArcGIS Online. Accessed August 25, 2021 at: https:// www.arcgis.com

19 Centers for Disease Control and Prevention. What is health literacy? Accessed August 25, 2021 at: https://www.cdc.gov/ healthliteracy/learn/index.html

20 National Library of Medicine. An introduction to health literacy. Accessed August 25, 2021 at: https://nnlm.gov/initiatives/topics/health-literacy

21 Centers for Disease Control and Prevention. http://www.cdc.gov/ 
22 Finset A. Challenges for healthcare communication during the COVID-19 pandemic. Patient Educ Couns 2021;104(02):215-216

23 Khubchandani J, Sharma S, Price JH, Wiblishauser MJ, Sharma M, Webb FJ. COVID-19 vaccination hesitancy in the united states: a rapid national assessment. J Community Health 2021;46(02):270-277

24 Jaiswal J, LoSchiavo C, Perlman DC. Disinformation, misinformation and inequality-driven mistrust in the time of COVID-19: lessons unlearned from AIDS denialism. AIDS Behav 2020;24(10):2776-2780

25 Doan S. Misrepresenting COVID-19: lying with charts during the second golden age of data design. J Bus Tech Commun 2021;35(01):73-79
26 Vaccine Hesitancy for COVID-19: State, County, and Local Estimates. Accessed May 15, 2021 at: https://aspe.hhs.gov/reports/vaccinehesitancy-covid-19-state-county-local-estimates

27 Andreotti A, Mingione E, Polizzi E. Local welfare systems: a challenge for social cohesion. Urban Stud 2021;49(09): 1925-1940

28 Allard SW. Places in Need: The Changing Geography of Poverty. New York: Russell Sage Foundation; 2017

29 PolicyMap. Predominant Racial or Ethnic Group map. Available at: https://www.policymap.com/ 